Artigo

\title{
Avaliação do Desempenho das Simulações por Conjunto do Modelo Eta-5km para o Caso de Chuva Intensa na Bacia do Rio Paraíba do Sul em Janeiro de 2000
}

\author{
Renata Novaes Calado ${ }^{1}\left[\mathrm{D}\right.$, , Claudine Pereira Dereczynski ${ }^{1}$, Sin Chan $\mathrm{Chou}^{2}$, Gustavo Sueiro ${ }^{2}$, \\ José Davi de Oliveira Moura ${ }^{2}$, Victor Rander da Silva Santos ${ }^{1}$ \\ ${ }^{I}$ Departamento de Meteorologia, Instituto de Geociências, Centro de Ciências Matemáticas \\ e da Natureza, Universidade Federal do Rio de Janeiro, Rio de Janeiro, RJ, Brasil. \\ ${ }^{2}$ Centro de Previsão do Tempo e Estudos Climáticos, Instituto Nacional de Pesquisas Espaciais, \\ Cachoeira Paulista, SP, Brasil.
}

Recebido em 28 de Março de 2017 - Aceito em 6 de Setembro de 2017

\begin{abstract}
Resumo
O objetivo deste estudo é avaliar o desempenho das simulações por conjunto do modelo Eta-5km do Instituto Nacional de Pesquisas Espaciais para um caso de chuva intensa ocorrido na Bacia do Rio Paraíba do Sul em janeiro de 2000, durante um evento de Zona de Convergência do Atlântico Sul. O conjunto de simulações é formado por 5 membros, denominados de acordo com suas parametrizações convectiva (Betts-Miller-Janjic - BMJ ou Kain-Fritsch - KF) e de microfísica (Ferrier ou Zhao): CNTRL (BMJ/Ferrier); KF (KF/Ferrier); KFMX (KF com fluxo de momentum/Ferrier); KFMXP (KF com fluxo de momentum e força do gradiente da perturbação da pressão/Ferrier) e ZHAO (BMJ/Zhao). As simulações são realizadas com horizontes de 24 até $120 \mathrm{~h}$ e condições de contorno inicial e lateral provenientes do "Climate Forecast System Reanalysis". Os resultados indicam que todos os membros são capazes de posicionar a banda de chuva aproximadamente na mesma localização observada. O modelo, apesar de subestimar os valores das maiores pluviometrias diárias, simula uma frequência maior de eventos de chuva forte. Em geral, a média do conjunto apresenta melhor performance do que cada um dos membros isoladamente e as simulações com $48 \mathrm{~h}$ de antecedência são superiores às demais.
\end{abstract}

Palavras-chave: simulações, precipitação, modelo Eta, alta resolução, parametrizações convectiva e de microfísica.

\section{Evaluation of Eta Model 5-km Ensemble Simulations of an Extreme Rainfall Event over the Paraíba do Sul River Basin During January 2000}

\begin{abstract}
The aim of this study is to evaluate the performance of simulations generated by the ensemble of the Eta-5km model from the National Institute for Space Research. The evaluation was performed over the Paraíba do Sul River Basin during an extreme rainfall event, occurred early in January 2000, in a South Atlantic Convergence Zone event. The 5-member ensemble is formed with different convective (Betts-Miller-Jancic - BMJ or Kain-Fritsch - KF) and microphysics (Ferrier or Zhao) parameterizations and are nominated respectively as: CNTRL (Betts-MillerJanjic/Ferrier); KF (Kain-Fritsch/Ferrier); KFMX (Kain-Fritsch with inclusion of momentum flux/Ferrier); KFMXP (Kain-Fritsch with inclusion of momentum flux and force of the perturbation of pressure gradient/Ferrier) and ZHAO (Betts-Miller-Janjic/Zhao). The simulations are integrated up to $120 \mathrm{~h}$ and the initial and boundary conditions were taken from the Climate Forecast System Reanalysis. The results indicate that all members were able to position the rain band in about the same observed location. Although the model underestimates the highest daily precipitation amount, it simulates higher frequency of heavy rainfall events than it really occurs. In general, the ensemble mean simulation presents higher performance than each individual members and the 48-h simulations are better than the other forecast lead times.
\end{abstract}

Keywords: simulations, rainfall, Eta model, high resolution, convective and microphysics parameterizations.

Autor de correspondência: Renata Novaes Calado, renatacalado@gmail.com. 


\section{Introdução}

Entre as diversas variáveis meteorológicas, a precipitação é uma das mais importantes. Ela interfere diretamente tanto em ações simples e rotineiras da população, quando ocorre com intensidade fraca a moderada, quanto deflagrando deslizamentos, inundações, alagamentos e outros desastres quando ocorre com intensidade forte e em eventos de longa duração. Nesses casos extremos, as consequências podem ser drásticas para as regiões afetadas, com mortos, feridos, grandes prejuízos econômicos e sociais. Embora a chuva seja uma das variáveis meteorológicas que mais impactam na vida cotidiana, os modelos numéricos ainda não são capazes de prever sua localização e intensidade com exatidão. Ademais, eventos de chuva extrema acrescentam maior dificuldade ao prognóstico, pois podem estar relacionados a padrões atmosféricos de baixa previsibilidade e podem afetar regiões de forma isolada e não generalizada.

As principais dificuldades relacionadas à previsão de eventos de chuvas intensas estão associadas à previsão quantitativa da precipitação e de sua localização. Muitas vezes erros de poucos quilômetros na localização da chuva podem significar a diferença entre um município e outro.

Os modelos globais de previsão numérica de tempo (PNT) têm mostrado bom desempenho para representar fenômenos meteorológicos em grande escala, contudo são limitados no que diz respeito à previsão de sistemas na meso e na micro escalas (Giorgi, 1990; 2006; Giorgi e Mearns, 1991). Os modelos regionais de PNT, com uma grade suficientemente fina, podem representar melhor os aspectos da camada limite, tais como topografia, vegetação, solos e o litoral (Feser et al., 2011; Solman, 2013). Além disso, tais modelos representam de forma mais adequada os sistemas meteorológicos na meso e micro escalas. Tanto nos modelos globais quanto nos regionais a precipitação precisa ser parametrizada pois os processos físicos envolvidos ocorrem em escalas menores do que as escalas envolvidas pelos modelos, e dessa forma não podem ser resolvidos explicitamente. A parametrização de precipitação prevista pelos modelos é em geral proveniente de esquemas de parametrização de convecção cumulus (ou parametrização convectiva), juntamente com a microfísica de nuvens (Lopez, 2007).

As PNTs podem ser determinísticas ou probabilísticas. Nas previsões determinísticas elabora-se apenas uma integração do modelo, onde objetiva-se prever exatamente o valor da variável em um determinado ponto de grade, sem muitas opções para auxiliar na tomada de decisões. Por outro lado, nas previsões probabilísticas o mesmo modelo ou vários modelos de PNT são integrados diversas vezes, formando-se um conjunto de previsões, sendo possível prever a probabilidade de ocorrência de um certo evento, o que resulta em melhor suporte à tomada de decisão (Toth et al., 1997).
Nas previsões probabilísticas são construídos conjuntos que consideram as incertezas nas condições iniciais e de contorno dos modelos e também nas parametrizações utilizadas para modelar processos atmosféricos. Tais imprecisões na descrição do estado inicial da atmosfera, juntamente com a dificuldade matemática de equacionar a dinâmica atmosférica, tendem a ser minimizadas com a técnica de previsão por conjunto (Palmer, 2000). Sendo assim, além de gerar previsão com o membro padrão, dito membro Controle, outros membros são gerados a partir de pequenas perturbações iniciais e/ou de contorno ou a partir de diferentes parametrizações. As informações obtidas através da previsão por conjunto devem ser tratadas por alguma metodologia de decisão ou algoritmos de pós-processamento e visualização (Calvetti, 2011).

O desenvolvimento da previsão por conjunto baseado em diferentes esquemas de produção de chuva e nuvem no modelo Eta mostrou-se bastante eficaz para a previsão de enchentes na bacia do rio Taquari-Antas (Siqueira et al., 2016).

Após a construção de um conjunto de previsões é necessário que haja uma avaliação para verificar se a construção foi adequada, assim como para identificar possíveis melhorias e aperfeiçoamento do conjunto. Diversas métricas de verificação da previsão são utilizadas de forma a, objetivamente, ponderar sobre a qualidade do prognóstico gerado. A partir dessa avaliação é possível verificar quais parametrizações contemplam melhor cada tipo de fenômeno meteorológico em cada região do domínio do modelo e assim obter um conjunto equilibrado.

A verificação de previsões por conjunto geralmente se dá de duas formas: a avaliação diagnóstica das características da distribuição do conjunto e a verificação das previsões de probabilidade derivadas do conjunto (Wilson et al., 1999).

Peel e Wilson (1999) realizaram uma criteriosa investigação sobre o conjunto canadense, avaliando a qualidade das previsões de chuva tanto para períodos secos quanto para períodos úmidos. Hamill e Colucci (1998), avaliaram um conjunto do modelo Eta-Regional Spectral Model (RSM) sobre o território Norte Americano e propuseram correções do tipo distribuição-gama para todas as variáveis, exceto a precipitação.

Tennessong et al. (2012) avaliaram o modelo Eta sobre a África central, confrontando as previsões geradas a partir de diferentes esquemas de parametrização da convecção: Kain-Fritsch (KF - Kain e Fritsch, 1993) e BettsMiller-Janjic (BMJ - Janjic, 1994) com dados do "Tropical Rainfall Measuring Mission” (TRMM). Seus resultados mostram que, apesar da temperatura do ar e da pressão ao nível médio do mar terem sido bem previstas, o modelo não foi capaz de prever o máximo de precipitação observada (cerca de $80 \mathrm{~mm} / 6 \mathrm{~h}$ ).

Gallus (1999) mostra que o esquema de parametrização BMJ apresentou melhores resultados em relação à 
localização de eventos extremos de chuva, quando comparado ao esquema KF. Através de simulações de casos de inundações sobre os Estados Unidos, com o modelo Eta utilizando ambos esquemas em diferentes resoluções espaciais, o autor concluiu que em altas resoluções o esquema $\mathrm{KF}$ tende a prever maiores acumulados de chuva. Esse estudo mostrou que, nos eventos de precipitação extrema, o esquema BMJ simula melhor a localização espacial dos picos de chuva, subestimando os acumulados, enquanto que o esquema $\mathrm{KF}$ desloca em diversos quilômetros o máximo de chuva, porém simula melhor sua intensidade.

Neste trabalho são avaliadas simulações por conjunto geradas pelo modelo Eta do Centro de Previsão de Tempo e Estudos Climáticos (CPTEC) do Instituto Nacional de Pesquisas Espaciais (INPE), para um evento extremo ocorrido na Bacia do Rio Paraíba do Sul (BRPS) no início de janeiro de 2000.

$\mathrm{O}$ caso estudado neste trabalho, descrito em detalhe em Brasiliense (2016) e Brasiliense et al. (2017) ocorreu entre os dias 1 e 5 de janeiro de 2000, provocando inundações e deslizamentos que deixaram 12 óbitos e mais de 6000 desabrigados, paralisando cerca de 11 quilômetros da Rodovia Presidente Dutra, que liga as duas maiores metrópoles do Brasil. De acordo com Sugahara et al. (2010), considerando-se o total pluviométrico acumulado em 5 dias, este evento de janeiro de 2000 pode ser classificado como muito raro, com probabilidade de ocorrência em um ano variando entre $0,0086 \%$ e $2,3 \%$.

O objetivo principal deste estudo é avaliar o desempenho da previsão por conjunto em alta resolução para prever eventos de chuva intensa na BRPS. A previsão por conjunto é construída a partir das diferentes representações de processos de produção de chuva no modelo Eta- $5 \mathrm{~km}$, totalizando-se cinco membros com horizontes de 24, 48, 72,96 e 120 h. São comparadas as performances das integrações do modelo que levam em conta as diferentes parametrizações convectivas e de microfísica, avaliadas no caso de chuva intensa ocorrido na BRPS em janeiro de 2000.

Na próxima seção apresenta-se a metodologia e os dados utilizados. Os resultados da avaliação das simulações são apresentados na seção 3. As conclusões e considerações finais são descritas na seção 4 .

\section{Metodologia e Dados}

A metodologia é apresentada da seguinte forma: inicialmente são descritas as características do modelo Eta e os membros do conjunto, em seguida são apresentadas as métricas de avaliação, e por último os dados utilizados para a avaliação.

\subsection{O Modelo Eta}

O modelo Eta é um modelo atmosférico desenvolvido em conjunto pela Universidade de Belgrado e pelo Instituto de Hidrometeorologia da antiga Iugoslávia e utilizado para propósitos operacionais e de pesquisa (Mesinger et al.,
1988). Sua principal característica é o uso da coordenada vertical eta (Mesinger, 1984), que resulta em uma coordenada de superfície quasi-horizontal, e reduz erros no gradiente de pressão devido à topografia. Tal característica é bastante vantajosa em localidades de topografia íngreme e complexa, como a área de estudo deste trabalho. O modelo Eta, que utiliza pontos de grade do tipo E de Arakawa, é utilizado no Brasil desde 1996 (Chou, 1996). Atualizações foram introduzidas no modelo, de forma que hoje é um modelo em volume finito, com refinamento adicional na coordenada vertical, que utiliza o esquema "piecewise linear" para advecção vertical, não-hidrostático, entre outras modificações descritas em Mesinger et al. (2012). A integração do modelo é explicita em dois níveis no tempo. $\mathrm{O}$ esquema de radiação resolve as ondas curtas e as ondas longas a partir de um pacote desenvolvido pelo GFDL (Geophysical Fluid Dynamics Laboratory) (Lacis e Hansen, 1974; Schwarzkopf e Fels, 1991). A superfície continental é resolvida pelo esquema NOAH (Ek et al., 2003) que distingue 13 tipos de cobertura vegetal e 18 tipos de solo, atualizado por Doyle et al. (2013).

Neste estudo são utilizadas simulações com grade horizontal de $5 \mathrm{~km}$ e 50 camadas verticais. As condições iniciais e de contorno lateral são provenientes do "Climate Forecast System Reanalysis", do "National Centers for Environmental Prediction" (CFSR/NCEP) (Saha et al., 2010), com $45 \mathrm{~km}$ de resolução horizontal. A condição de contorno lateral é atualizada a intervalos de $6 \mathrm{~h}$. Diferente dos demais modelos regionais, o modelo Eta não utiliza a condição de contorno lateral de relaxação. A condição do Eta é resolvida como um problema matemático de condição de contorno (Mesinger, 1977). A comparação entre os dois métodos de condição de contorno lateral, mostra previsão com melhor desempenho utilizando o método de Mesinger (Mesinger e Veljovic, 2013).

Aninhamentos consecutivos não são necessários no modelo Eta, conforme apresentado em Rozante (2001). O domínio grande para o modelo Eta é vantajoso porque permite espaço para que o modelo regional desenvolva a estrutura de escala mais fina que a condição da forçante lateral. Esse é o papel de um modelo de mesoescala, fornecer estrutura mais fina que a da forçante do contorno lateral. Portanto, o modelo Eta não utiliza "nudging" interno para desenvolver livremente a estrutura fina. O trabalho de Mesinger e Veljovic (2017) mostra o ganho do desempenho da previsão do modelo Eta sobre as previsões do modelo do "European Centre for Medium Range Weather Forecast" (ECMWF) utilizadas no contorno lateral.

A precipitação no modelo Eta é produzida de forma implícita a partir da parametrização de convecção cumulus e a explícita a partir da parametrização de microfísica de nuvens. A chuva da microfísica de nuvens é gerada a partir da saturação do ar, não sendo condicionada à instabilidade convectiva. Por outro lado, a precipitação convectiva é gerada por cumulus e cumulonimbus e está relacionada à 
massas de ar convectivamente instáveis. Diferentes tipos de precipitação geram distintos perfis de calor latente na atmosfera, o que implica em diferentes resultados nas escalas de tempo e clima.

\subsection{Membros do conjunto}

Os membros do conjunto da previsão são construídos a partir de diferentes representações dos processos físicos, em particular a produção de chuva do modelo Eta. São utilizados os esquemas de convecção Betts-Miller-Janjic (Janjic, 1994); Kain-Fritsch (Kain, 2004); Kain-Fritsch com fluxo de momentum (Carvalho, 2007) e Kain-Fritsch com fluxo de momentum e gradiente da perturbação da pressão (Moura, 2016), e os esquemas de microfísica de nuvens Ferrier (Ferrier, 2002; Ferrier 1994) e Zhao (Zhao et al., 1997).

A parametrização de convecção cumulus BMJ é um tipo de esquema de ajuste convectivo baseado no conceito de "lapse-rate" atmosférico. Essa parametrização utiliza um perfil termodinâmico de referência para representar a estrutura vertical em situações convectivas. O perfil de referência é ajustado de acordo com três parâmetros: déficit de pressão de saturação (determina o perfil de umidade); coeficiente de estabilidade (determina o perfil da temperatura) e o tempo do ajuste convectivo.

A parametrização de convecção cúmulos KF é um esquema do tipo fluxo de massa lagrangeano, que simula o entranhamento e detranhamento nas correntes convectivas para permitir uma interação entre a nuvem e o ambiente. A parametrização $\mathrm{KF}$ possui três partes fundamentais: i) $\mathrm{A}$ função de gatilho, que identifica as camadas com potencial convectivo e calcula a flutuabilidade da parcela, os efeitos de entranhamento e detranhamento; ii) A formulação do fluxo de massa, que representa os fluxos convectivos e iii) $\mathrm{O}$ fechamento, que redistribui a massa na coluna, removendo a energia potencial inicial disponível para a convecção.

Nas integrações realizadas para este estudo foi incluído, nos membros com parametrização KF, um parâmetro para controlar o quanto de água líquida/gelo será convertido em precipitação e o quanto será retido no ponto de grade do modelo, conforme proposto por Gomes e Chou (2010). Essa modificação é feita com o intuito de se ajustar a produção de precipitação em mesoescala.

O membro KFMX proposto por Carvalho (2007), resulta da inclusão do transporte de momentum convectivo no membro KF. Tal modificação considera os efeitos das correntes ascendente e descendente com entranhamento e detranhamento, que permitem a interação entre a nuvem e o ambiente, conservando massa, calor e momentum. Carvalho (2007) ressalta que tal modificação intensifica a vorticidade ciclônica em baixos níveis e a geração de regiões com vorticidade anticiclônica em altos níveis, promovendo o desenvolvimento de áreas de atividade convectiva profunda. Dessa forma, no campo de precipitação, são esperados acumulados mais intensos em situações pré-frontais.
O membro KFMXP possui o transporte de momentum convectivo e a força do gradiente de perturbação da pressão na escala da nuvem, conforme sugerido em Moura (2016). Essa modificação visa refinar os efeitos de transporte de momentum devido a perturbação da pressão, que normalmente é desprezada em modelos de parcela.

Os membros KF, KFMX e KFMXP utilizam o esquema de microfísica de nuvens de Ferrier (Ferrier et al., 2002). Esse esquema foi formulado para prever a variação de seis formas da água, representando assim a nuvem e a chuva da escala de grade do modelo. Além disso, o esquema considera diversos processos microfísicos da água na nuvem com interações entre as partículas de água da nuvem, cristais de gelo e água precipitável. O esquema prevê mudanças na umidade específica do vapor d'água e possui algoritmos de condensação do vapor e fluxo de precipitação e armazenamento da água na nuvem. Essa parametrização gera precipitação na forma de chuva, chuva congelada e gelo, levando em consideração os processos de mistura de fases, congelamento e derretimento.

O membro ZHAO utiliza o esquema de microfísica de nuvens Zhao (Zhao et al., 1997). Esse esquema apresenta como característica principal a inclusão da previsão de conteúdo de gelo ou água na nuvem com uma única variável preditiva, a taxa de mistura, que representa ambos. Dessa forma, o conteúdo de vapor d'água na nuvem, que envolve a formação e evaporação de nuvens e de precipitação, é calculado de forma explícita. Esse método permite uma melhor representação dos efeitos termodinâmicos de condensação em subgrade (precipitação e não precipitação), assim como uma relação mais estreita entre os processos radiativos, dinâmicos e hidrológicos no modelo.

As simulações são integradas para um horizonte de até $120 \mathrm{~h}$, em um domínio que cobre as Regiões Sul e Sudeste do Brasil, estendendo-se de $34^{\circ} \mathrm{S}$ a $12^{\circ} \mathrm{S}$ e de $59^{\circ}$ $\mathrm{O}$ a $36^{\circ} \mathrm{O}$.

A identificação de cada membro do conjunto de simulações com os esquemas de convecção e de microfísica é resumida na Tabela 1.

Tabela 1 - Parametrizações utilizadas por cada membro do conjunto de simulações com modelo Eta-5km.

\begin{tabular}{lll}
\hline \multirow{2}{*}{ Membro } & \multicolumn{2}{c}{ Parametrizações } \\
\cline { 2 - 3 } & Convecção cumulus & Microfísica de nuvens \\
\hline CNTRL & Betts-Miller-Janjic & Ferrier \\
KF & Kain-Fritsch & Ferrier \\
KFMX & $\begin{array}{l}\text { Kain-Fritsch com inclusão de } \\
\text { fluxo de momentum }\end{array}$ & Ferrier \\
KFMXP & $\begin{array}{l}\text { Kain-Fritsch com inclusão do } \\
\text { fluxo de momentum e força do } \\
\text { gradiente de pressão da nuvem }\end{array}$ & Ferrier \\
ZHAO & Betts-Miller-Janjic & Zhao \\
\hline
\end{tabular}




\subsection{Métricas de avaliação}

Duas formas distintas de avaliação são empregadas. A primeira, uma avaliação subjetiva, feita de forma visual, onde os campos de precipitação gerados pelo conjunto e por cada membro isolado são comparados ao campo de precipitação observada. Essa comparação é feita em diferentes datas e com diferentes horizontes de previsão. Somando-se a essa avaliação, são geradas evoluções temporais da precipitação (observada e prevista) para as localidades onde foram registrados os maiores acumulados de precipitação. Dessa forma, visa-se avaliar localmente o desempenho do modelo.

A segunda forma consiste de uma avaliação objetiva categórica. As métricas usadas para a verificação objetiva, assim como definidas em Mesinger e Black (1992), consistem do BIAS dado pela Eq. (1) e do Índice crítico de sucesso equitativo (ou "Equitable Threat Score" - ETS) apresentado na Eq. (2).

$$
\begin{aligned}
& B I A S=\frac{F}{O} \\
& \text { ETS }=\frac{H-C H}{F+O-H-C H}
\end{aligned}
$$

onde, para cada limiar de precipitação, $F$ é o número de eventos previstos, $O$ o número de eventos observados, $H$ o número de acertos e $\mathrm{CH}$ é o fator de correção dado pela Eq. (3):

$$
C H=\frac{F \times O}{N}
$$

onde $N$ representa o número total de eventos.

Em uma previsão perfeita o BIAS e o ETS apresentam valor 1. Para detalhar a avaliação, os eventos são subdivididos em categorias de acordo com o total pluviométrico diário, e assim, os índices são calculados para cada uma das categorias: i) $\geq 0,3 \mathrm{~mm} / \mathrm{dia}$; ii) $\geq 10,0 \mathrm{~mm} / \mathrm{dia}$; iii) $\geq 20,0 \mathrm{~mm} / \mathrm{dia}$; iv) $\geq 30,0 \mathrm{~mm} / \mathrm{dia}$; v) $\geq 40,0 \mathrm{~mm} / \mathrm{dia}$; vi) $\geq 50,0 \mathrm{~mm} /$ dia; vii) $\geq 60,0 \mathrm{~mm} /$ dia viii) $\geq 70,0 \mathrm{~mm} /$ dia e ix) $\geq 80,0 \mathrm{~mm} / \mathrm{dia}$.

\subsection{Dados}

Para a avaliação subjetiva são construídos mapas de precipitação diária, a partir dos dados gerados por Rozante et al. (2010), por eles denominado Merge. Tal conjunto de dados, gerado para o período de 1998 a 2016, possui resolução temporal de 3 h e resolução espacial de $25 \mathrm{~km}$, e inclui dados observados de precipitação combinados com dados do TRMM para toda a América do Sul e oceano adjacente.

Para a avaliação objetiva são utilizados totais pluviométricos diários de 23 estações na região da BRPS. Dez Plataformas de Coleta de Dados do INPE, localizadas no Rio de Janeiro (Barra Mansa); em São Paulo (Cachoeira Paulista, Cruzeiro, Cunha, Guaratinguetá, Monteiro Lobato, Paraibuna, São José do Barreiro e Silveiras); e em
Minas Gerais (Leopoldina). Nove estações meteorológicas convencionais do Instituto Nacional de Meteorologia no Rio de Janeiro (Campos dos Goytacazes, Cordeiro, Itaperuna, Nova Friburgo e Resende), São Paulo (Taubaté) e Minas Gerais (Barbacena, Coronel Pacheco e Juiz de Fora). Três postos pluviométricos do Departamento de Águas e Energia Elétrica de São Paulo: Aparecida, Bananal e Pindamonhangaba (Pico do Itapeva) e uma estação meteorológica do Instituto Estadual do Ambiente, localizada em Paty do Alferes (RJ). A avaliação estende-se por um período de cinco dias, de 01 a $05 / 01 / 2000$. Considerando-se todos os horizontes de previsão ( 24 h, 48 h, 72 h, 96 h e 120 h) e os 5 membros, além da média do conjunto, obtém-se um total de 3450 pares de observação e simulação para comparação. Os totais pluviométricos diários são acumulados entre 1200 UTC do dia anterior até 1200 UTC do dia em questão.

\section{Resultados}

No caso de chuva intensa analisado neste trabalho a precipitação mais forte foi registrada entre 1200 UTC do dia 02 e 1200 UTC do dia 03/01/2000, como apresentado em Brasiliense (2016). Assim, apesar da avaliação ser feita para todo o período, de 01 a $05 / 01 / 2000$, as análises dos dias 02 e 03/01/2000 são destacadas.

\subsection{Avaliação subjetiva}

A avaliação subjetiva é elaborada confrontando-se os campos simulados pelos membros do modelo Eta- $5 \mathrm{~km}$ com 24 e $48 \mathrm{~h}$ de antecedência para os dias 2 e 3 de janeiro de 2000 com os campos observados nesses mesmos dias, gerados com o Merge. A Fig. 1 apresenta os campos de precipitação observados nos dias 2 e 3 de janeiro de 2000. As simulações com 24 e $48 \mathrm{~h}$ de antecedência, respectivamente para os dias 2 e 3 de janeiro de 2000, são apresentadas nas Figs. 2-5.

Para o dia 02/01/2000 (Fig. 1a) os maiores acumulados de precipitação do domínio ocorrem dentro da BRPS, próximo à divisa dos estados do Rio de Janeiro e São Paulo. Ao comparar as simulações de $24 \mathrm{~h}$ de antecedência (Fig. 2) com a observação, nota-se que os membros que melhor simulam a precipitação para este dia são CNTRL e ZHAO (ambos com parametrização convectiva BMJ), sendo que o CNTRL apresenta desempenho ligeiramente melhor. Os demais membros, que possuem parametrização convectiva $\mathrm{KF}$, simulam os máximos de chuva no continente um pouco mais ao norte do observado, além de acumulados mais elevados. Da mesma forma, ao analisar a distribuição da precipitação em todo o domínio, verifica-se que os membros com parametrização BMJ simulam melhor os totais de chuva, enquanto que os membros com parametrização KF os superestimam. Nota-se também que nas simulações com parametrização KF (em todas as datas analisadas) a chuva apresenta um aspecto granular diferente das demais parametrizações, onde a chuva distribui-se de forma mais homogênea. Esse fato ocorre, pois, diferente da parametri- 
(A) $02 / 01 / 2000$

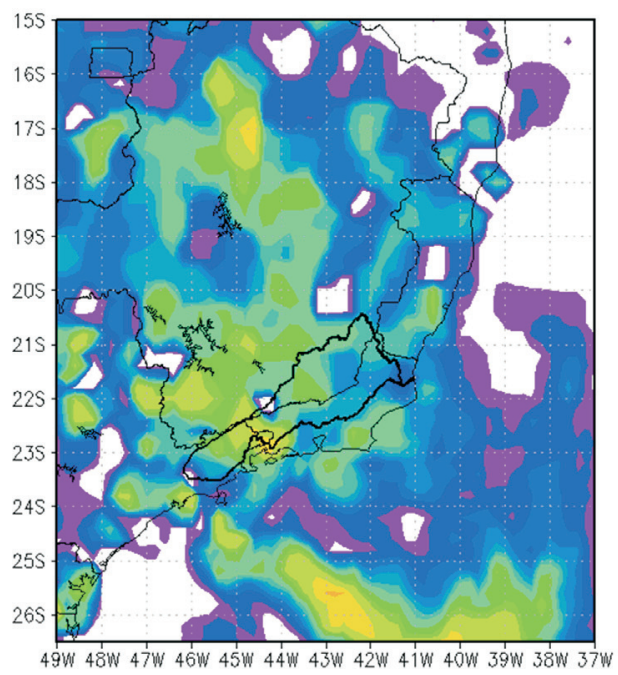

(B) $03 / 01 / 2000$

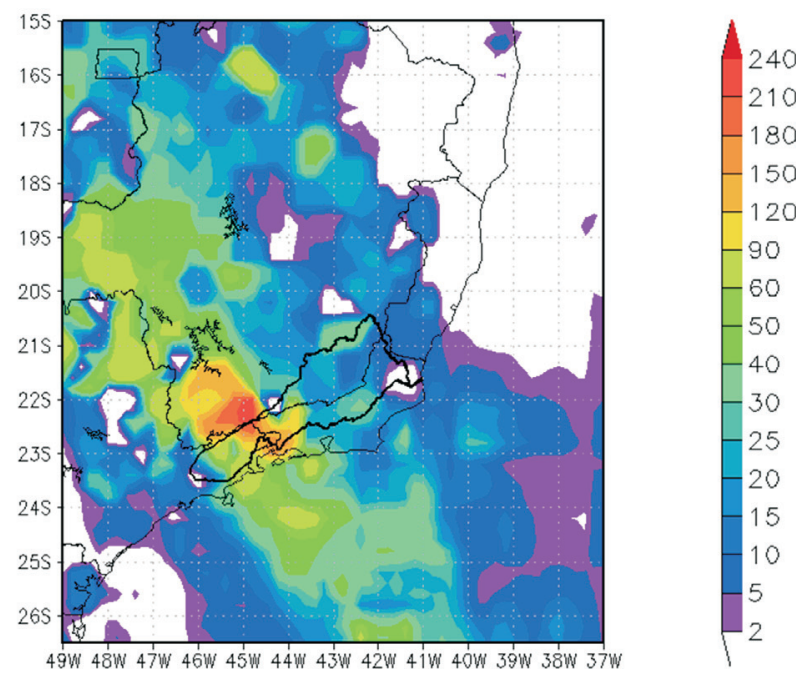

Figura 1 - Precipitação (mm/dia) observada em: (A) 02/01/2000 e (B) 03/01/2000.

(A) CNTRL

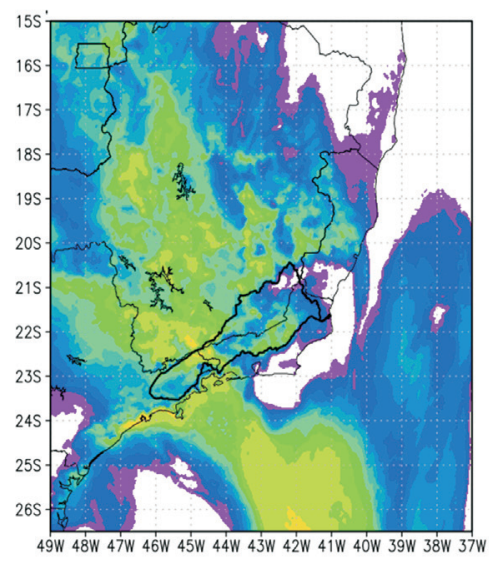

(D) KFMXP

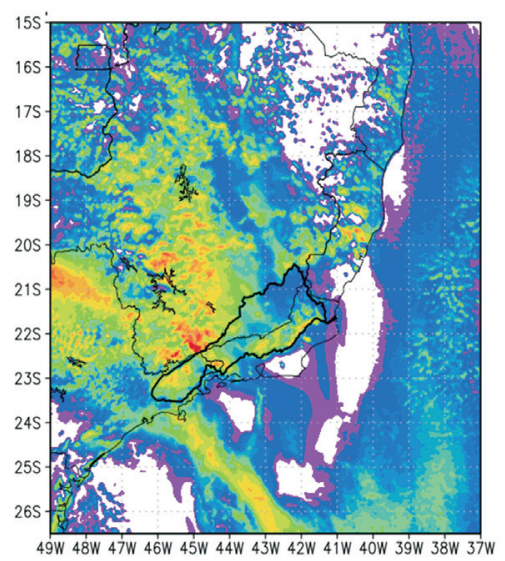

(B) $\mathrm{KF}$

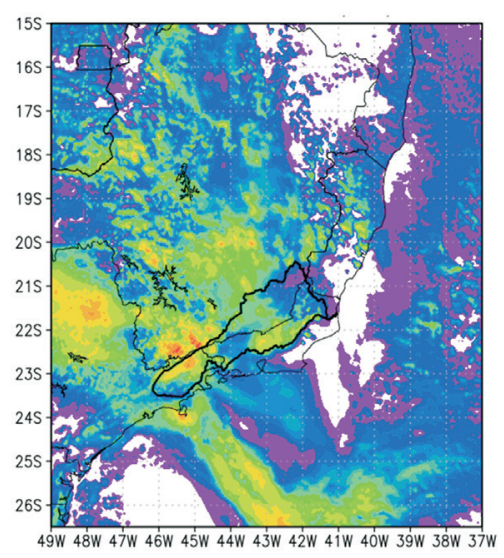

(E) ZHAO

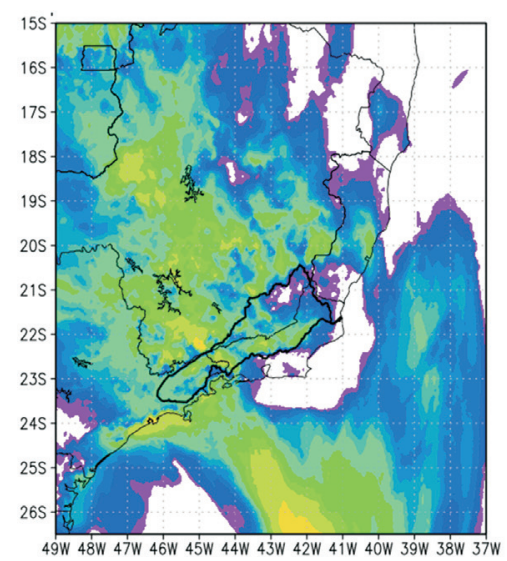

(C) KFMX

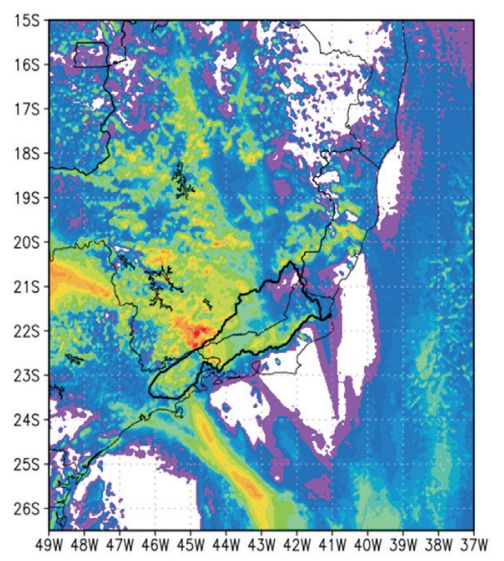

(F)Média Conjunto

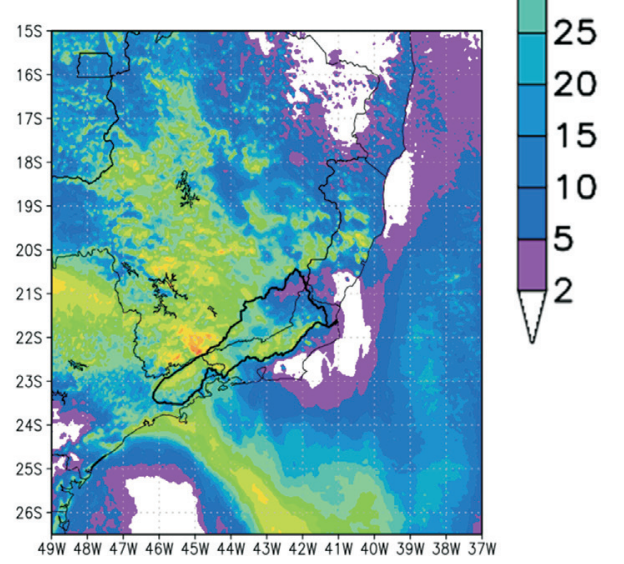

Figura 2 - Precipitação (mm/dia) simulada com 24 h de antecedência para o dia 02/01/2000 pelos membros: (A) CNTRL, (B) KF, (C) KFMX, (D) KFMXP, (E) ZHAO e (F) Média do conjunto. 
zação BMJ, que dispara a convecção de acordo com o padrão de grande escala, a parametrização $\mathrm{KF}$ inicia a convecção em escala local. Dessa forma, uma escala horizontal mais refinada, como a utilizada neste trabalho, gera chuva com tal aspecto "granulado". Analisando todo o domínio, observa-se uma banda de precipitação intensa com orientação NO-SE, desde o sul de Minas Gerais até o oceano, com um núcleo mais intenso na divisa entre RJ e MG (Fig. 1a). A média do conjunto (Fig. 2f) simula bem com $24 \mathrm{~h}$ de antecedência, o posicionamento dessa banda.

Ainda para o dia 02/01/2000, as simulações com $48 \mathrm{~h}$ de antecedência (Fig. 3) indicam que os membros KF, KFMX e KFMXP também superestimam os acumulados de chuva principalmente no continente, no sul de Minas Gerais, com destaque para o membro KFMXP que simula uma região de chuva intensa no sul de Minas Gerais e na Região Serrana do Rio de Janeiro, que não são observadas. Os membros CNTRL e ZHAO simulam melhor os totais pluviométricos, porém nota-se que nenhum dos membros é capaz de simular o núcleo de chuva ocorrido no noroeste de Minas Gerais.

No dia 03/01/2000 ocorrem os maiores valores de pluviometria na BRPS (Fig. 1 b), com um máximo observado in situ de $192 \mathrm{~mm} / 24 \mathrm{~h}$ em Cruzeiro (a simulação que mais se aproximou desse valor nesse ponto foi de $118 \mathrm{~mm} / 24 \mathrm{~h}$, pelo membro CNTRL com $48 \mathrm{~h}$ de antecedência). Ainda na Fig. 1b, nota-se uma região de máximo de precipitação no sul de Minas Gerais. Nas simulações com $24 \mathrm{~h}$ de antecedência para este dia (Fig. 4) todos os membros do conjunto foram capazes de posicionar um máximo de chuva aproximadamente na mesma localização do observado, porém todos os membros subestimam a intensidade da chuva. De maneira geral, também para esta data, os membros CNTRL e ZHAO apresentam os melhores desempenhos, pois ambos são mais acurados no posicionamento da banda de precipitação entre o sul de Minas Gerais e o Sul do estado do Rio de Janeiro. Os membros com parametrização Kain-Fritsch: KF, KFMX e KFMXP
(A) CNTRL

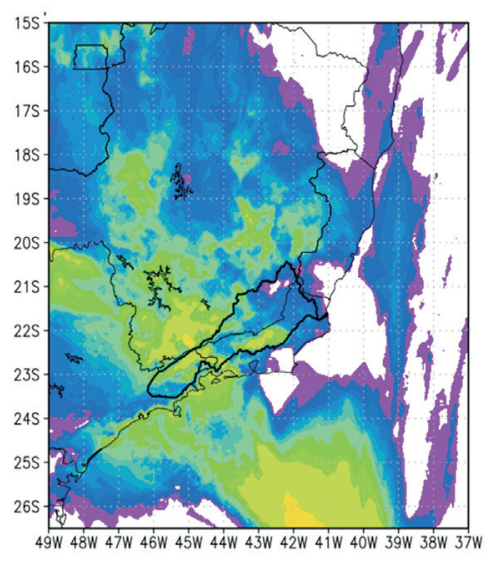

(D) KFMXP

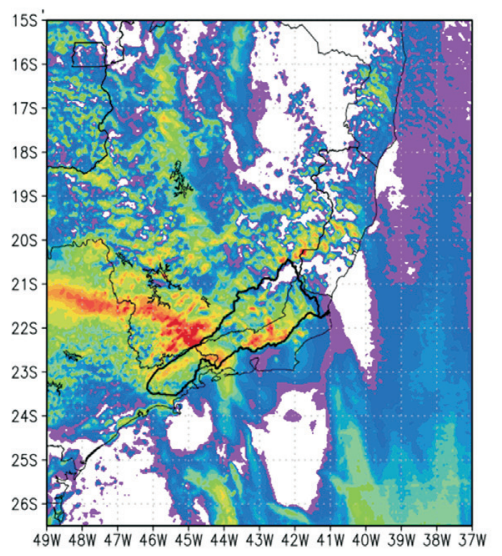

(B) KF

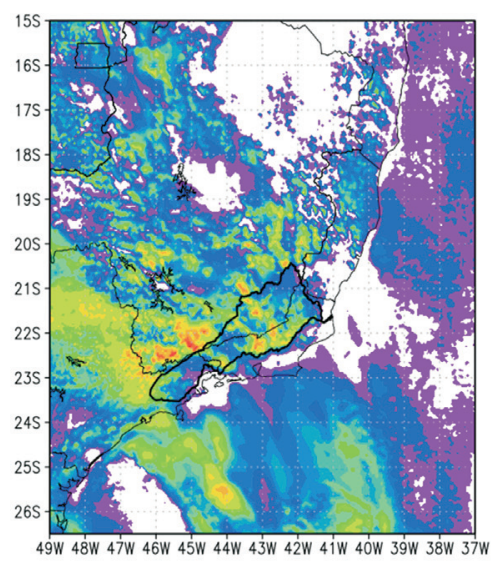

(E) ZHAO

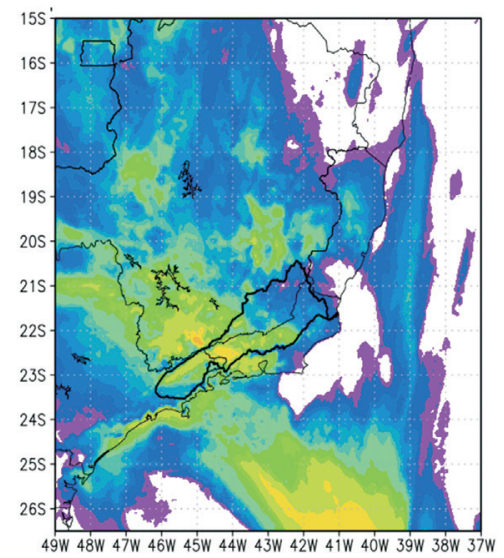

(C) KFMX

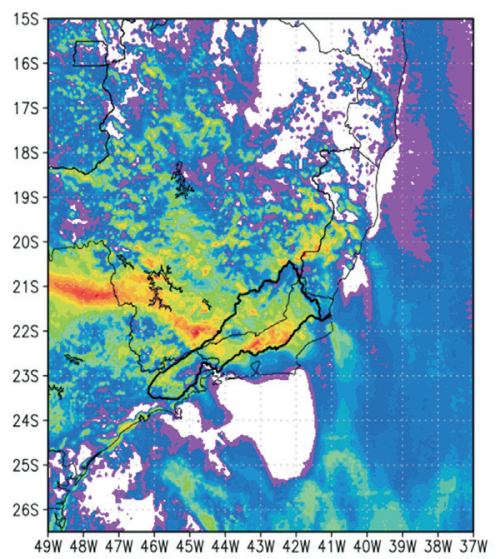

(F)Média Conjunto

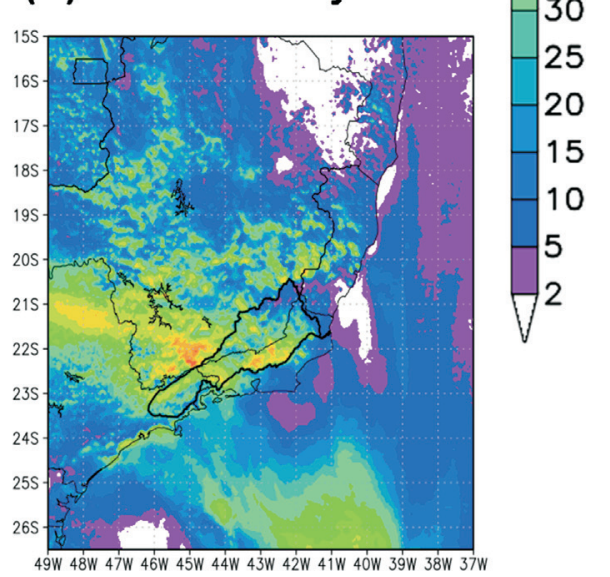

Figura 3 - Precipitação (mm/dia) simulada com 48 h de antecedência para o dia 02/01/2000 pelos membros: (A) CNTRL, (B) KF, (C) KFMX, (D) KFMXP, (E) ZHAO e (F) Média do conjunto. 
(A) CNTRL

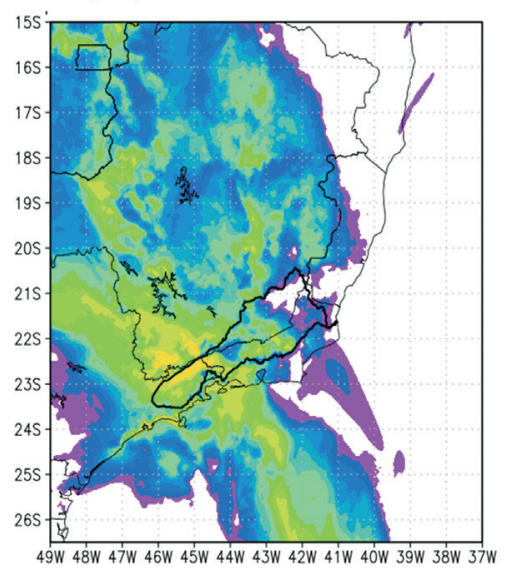

(D) KFMXP

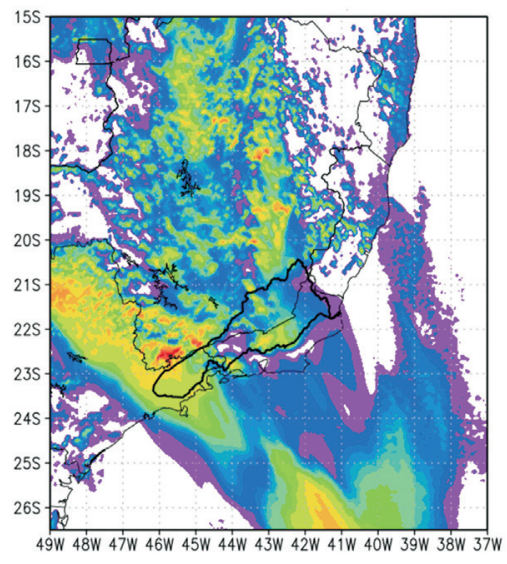

(B) KF

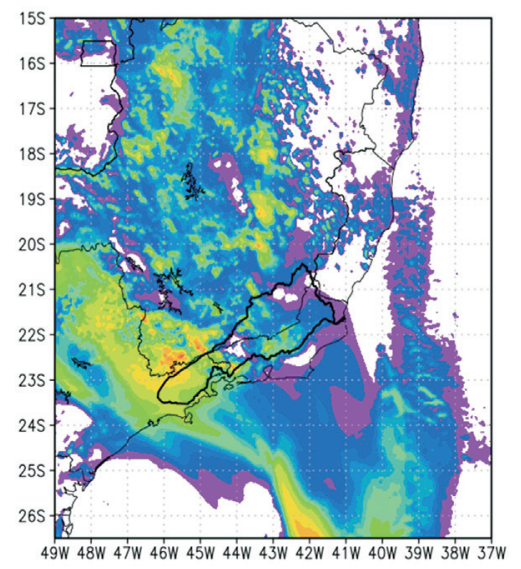

(E) ZHAO

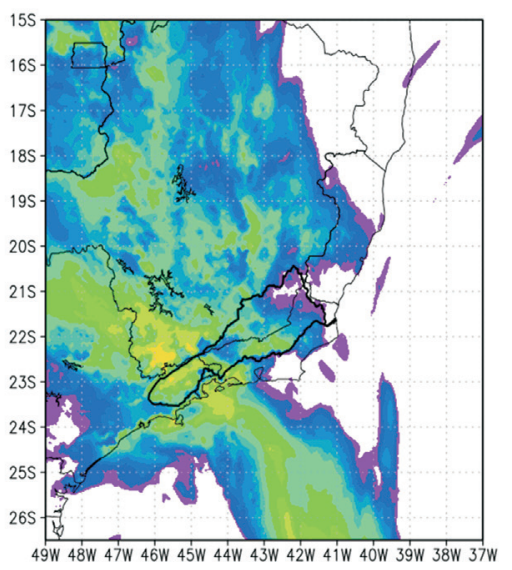

(C) KFMX

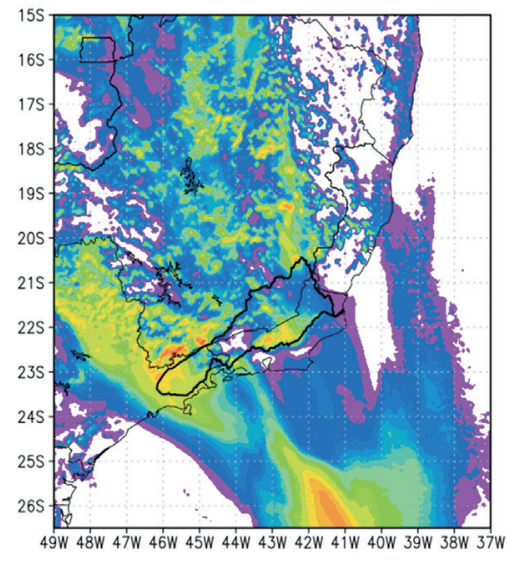

(F)Média Conjunto

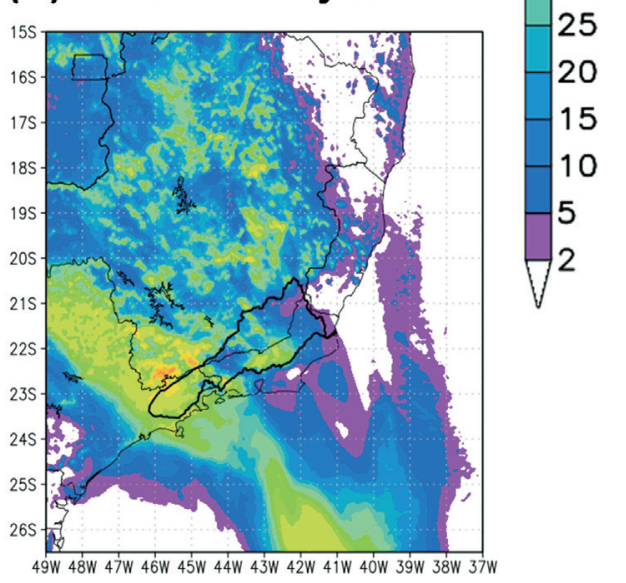

Figura 4 - Precipitação (mm/dia) simulada com 24 h de antecedência para o dia 03/01/2000 pelos membros: (A) CNTRL, (B) KF, (C) KFMX, (D) KFMXP, (E) ZHAO e (F) Média do conjunto.

posicionam a banda de precipitação ligeiramente mais ao sul, atrasada em relação à observação.

Finalmente as simulações para o dia 03/01/2000 com horizonte de 48 h são apresentadas na Fig. 5. Todos os membros do conjunto são capazes de posicionar a banda de precipitação aproximadamente na mesma região em que foi observada, o que demonstra um ganho de qualidade em relação à simulação com $24 \mathrm{~h}$ de antecedência. Esse melhor desempenho das simulações de $48 \mathrm{~h}$ sobre as de $24 \mathrm{~h}$ resulta da condição inicial ser proveniente da reanálise do CFSR, no lugar de uma análise do próprio modelo Eta. Esse comportamento já foi constatado em estudos anteriores principalmente no padrão do BIAS (Chou e Justi, 1999; Bustamante et al., 1999; Bustamante et al., 2005). Nos instantes iniciais da integração, o modelo produz fortes ajustes e gera muita onda de gravidade. Algumas variáveis dos processos físicos de modelos iniciam com valores zerados, esse é o caso da precipitação que inicia com valores nulos. Os esquemas de produção de chuva necessitam de algumas chamadas até conseguirem acumular os totais de chuva a valores consistentes com as condições do ambiente de uma tempestade nos modelos numéricos.

Os membros KFMX e KFMXP simulam valores aproximados à observação na região de máxima precipitação, porém esses membros também simulam valores elevados de precipitação na Região Serrana do Rio de Janeiro e na região central de Minas Gerais, que de fato não ocorreram. A média do conjunto também apresenta boa performance para simular a localização e a intensidade da precipitação observada.

De maneira geral, as simulações geradas pelo membro CNTRL apresentam boa performance em ambas as datas. Para o dia 03/01/2000, as simulações com horizonte de $48 \mathrm{~h}$ se mostram superiores em todos os membros do conjunto, em comparação aos demais horizontes. Os membros KFMX e KFMXP apresentam desempenho mais acurado quando comparados ao membro KF, o que evidencia que a inclusão do fluxo de momento na parametrização alcança melhores resultados. A média do conjunto apresenta boa performance para simular a precipitação obser- 
(A) CNTRL

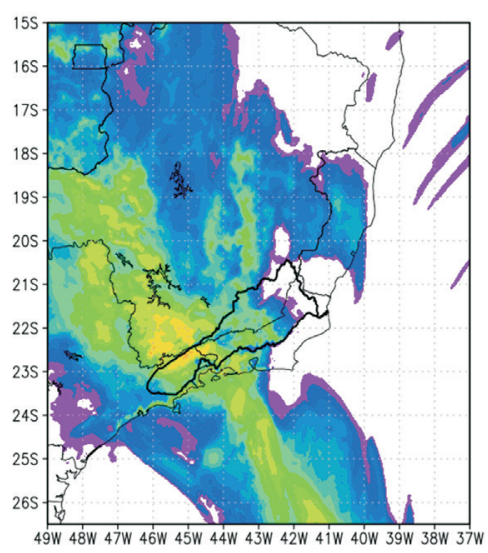

(D) KFMXP

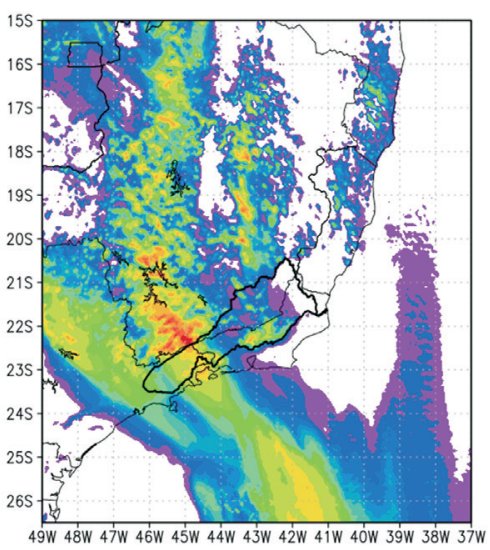

(B) KF

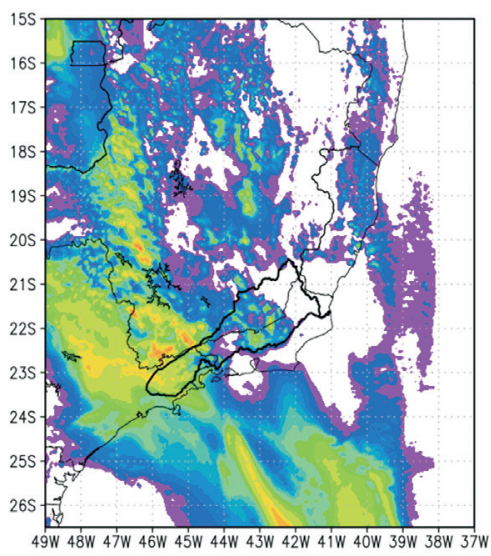

(E) ZHAO

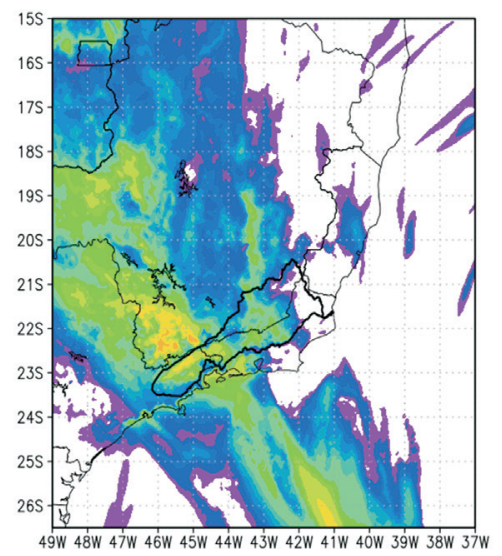

(C) KFMX

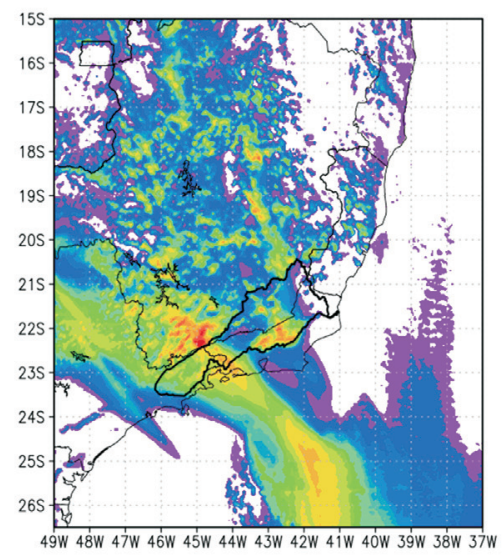

240

210

180

150

120

$-90$

60

50

40

30

(F)Média Conjunto

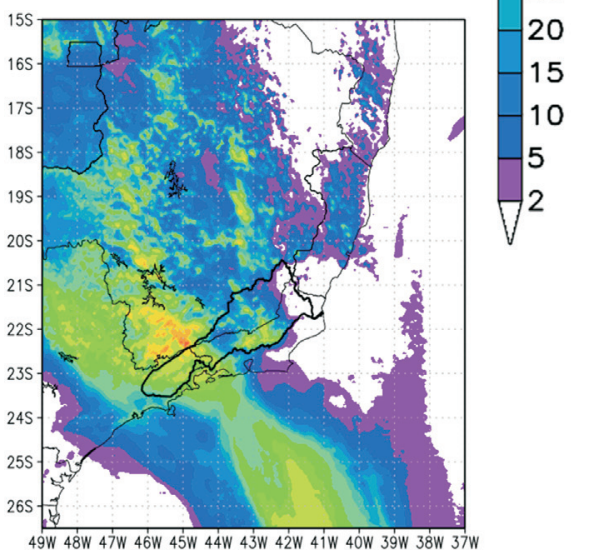

Figura 5 - Precipitação (mm/dia) simulada com 48 h de antecedência para o dia 03/01/2000 pelos membros: (A) CNTRL, (B) KF, (C) KFMX, (D) KFMXP, (E) ZHAO e (F) Média do conjunto.

vada, reduzindo a superestimativa característica dos membros com parametrização KF e sendo capaz de posicionar com bom desempenho a banda de precipitação, assim como os membros com parametrização BMJ.

Assim como nos resultados apresentados em Tennessong et al. (2012), neste trabalho o modelo Eta apresentou dificuldades em simular os valores extremos de precipitação observados. Ademais, os resultados aqui apresentados concordam com aqueles obtidos por Gallus (1999), onde os membros com parametrização BMJ localizaram melhor os máximos de chuva, porém os membros com parametrização KF se aproximaram mais da intensidade da pluviometria observada.

A Fig. 6 apresenta os totais pluviométricos acumulados entre 1200 UTC do dia 02/01/2000 e 1200 UTC do dia 03/01/2000 nos 23 postos pluviométricos localizados dentro da BRPS e também os valores simulados com $48 \mathrm{~h}$ de antecedência por cada um dos membros do conjunto e sua média. Observa-se que os dois maiores acumulados de chuva ocorreram em Cruzeiro (192 mm/dia) e Bananal (153 mm/dia), cujas simulações para todo o período são apresentadas na Fig. 7. Observa-se ainda na Fig. 6 que quando a precipitação é mais elevada, ocorre subestimativa em geral para todos os membros e também nesses casos, a dispersão entre os membros é maior. Além disso, o membro KF (KFMX) apresenta, em geral, os menores (maiores) totais pluviométricos, não ultrapassando $94 \mathrm{~mm}(154 \mathrm{~mm})$. Em geral, a média dos membros se aproxima mais das observações do que cada membro individualmente.

A Fig. 7 apresenta a evolução temporal da precipitação em Cruzeiro e Bananal, juntamente com os valores simulados por cada membro do conjunto com $48 \mathrm{~h}$ de antecedência. Em Cruzeiro (Fig. 7a), nota-se que a precipitação máxima observada ocorreu no dia 03/01/2000, e todos os membros foram capazes de prever o pico de chuva no dia $03 / 01 / 2000$, porém com menor intensidade, principalmente o membro KF, cuja precipitação simulada foi inferior a $40 \mathrm{~mm} / \mathrm{dia}$. O membro CNTRL apresenta melhor performance do que os demais, apesar de assim como os demais membros subestimar a precipitação observada em todo o período. Na cidade de Bananal, cuja evolução da chuva é mostrada na Fig. 7.b, nota-se um comportamento 


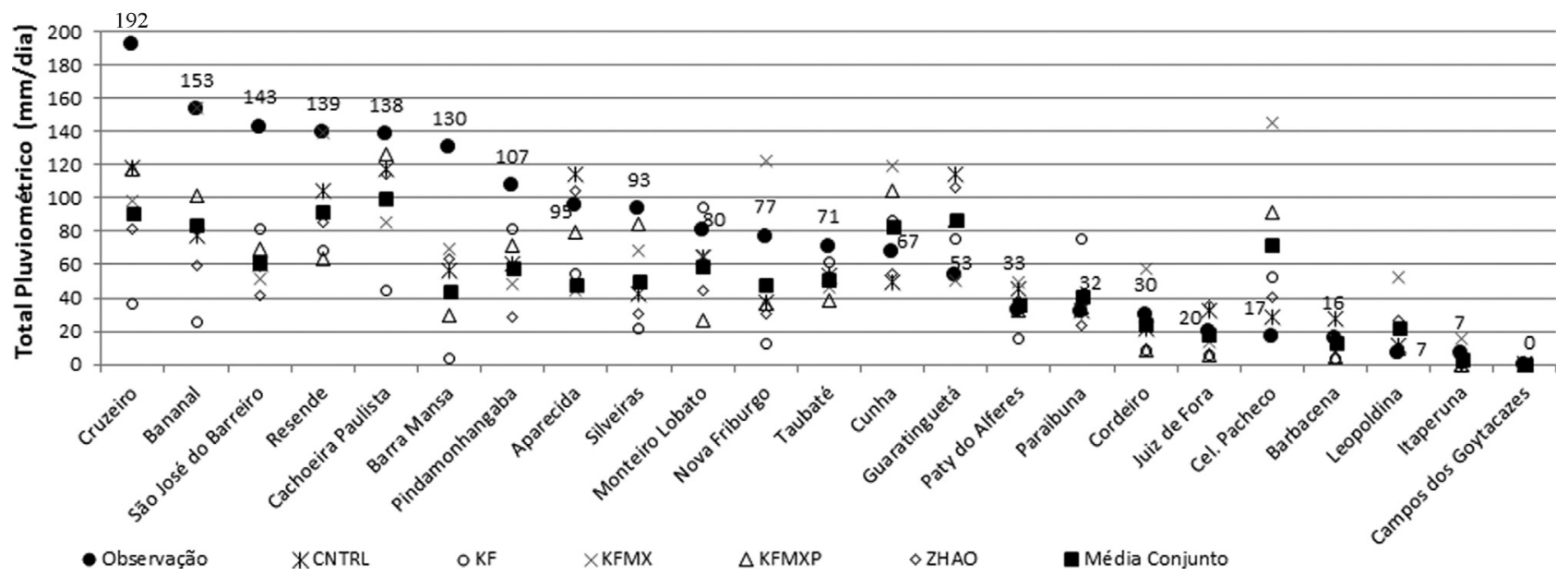

Figura 6 - Totais pluviométricos (mm/dia) observados e simulados com $48 \mathrm{~h}$ de antecedência para o dia 03/01/2000 pelos membros CNTRL, KF, KFMX, KFMXP, ZHAO e Média do conjunto nos 23 postos pluviométricos localizados dentro da Bacia do Rio Paraíba do Sul.

\section{(A) Cruzeiro 48h}

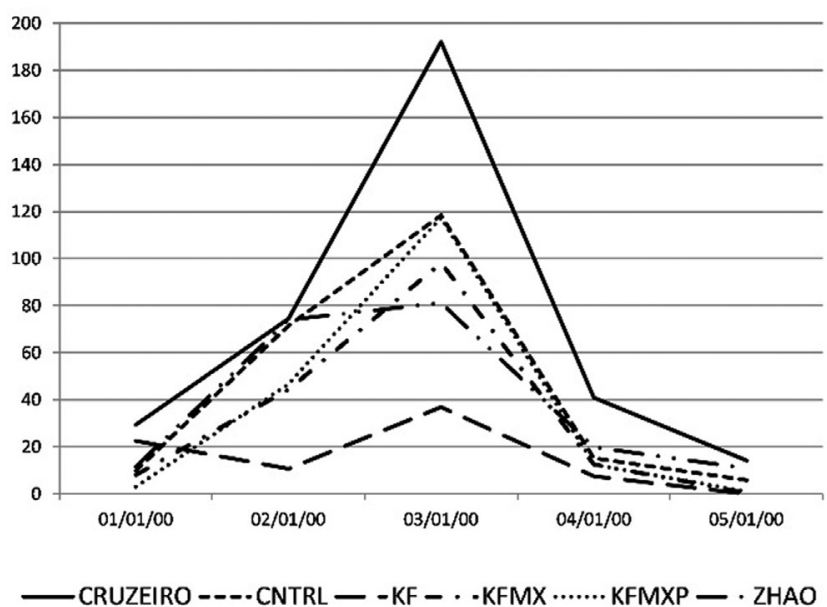

(B) Bananal 48h

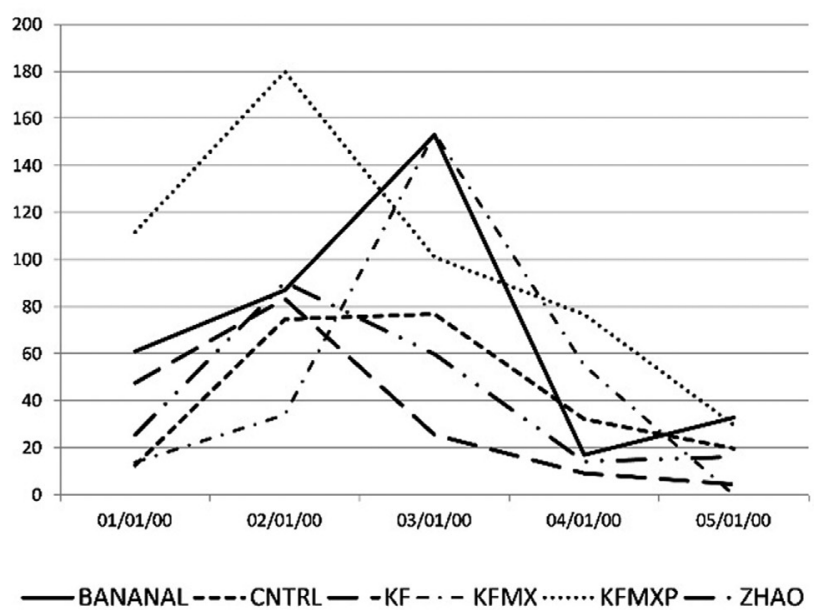

Figura 7 - Comparação da evolução da precipitação (mm/dia) observada (linha preta contínua) e simulada pelos membros CNTRL, KF, KFMX, KFMXP e ZHAO, em Cruzeiro (A) e Bananal (A) entre 01 e 05/01/2000.

ligeiramente diferente. O máximo de precipitação também ocorreu no dia 03/01/2000, porém, com exceção dos membros CNTRL e KFMX, o máximo foi previsto para o dia 02/01/2000. O Membro KFMXP simulou elevados valores para o dia $02 / 01 / 2000$, maiores que os observados no dia 03/01/2000. O membro KFMX apresentou a melhor performance nesta localidade, com valores aproximados dos observados no dia 03/01/2000.

\subsection{Análise objetiva}

As métricas de análise objetiva utilizadas são o BIAS e o ETS, ambas calculadas para um total de 3450 pares de observação e simulação de chuva, conforme descrito na metodologia. Neste trabalho são mostrados apenas os gráficos das métricas para $24 \mathrm{~h}$ e $48 \mathrm{~h}$, que se mostram mais acuradas do que as demais. Tal avaliação estatística foi aplicada para o período entre 01 e 05/01/2000.

A Fig. 8 apresenta os gráficos de ETS e BIAS para todos os membros incluindo a média do conjunto. Os cálculos são feitos para diferentes categorias de precipitação, desde chuva maior ou igual a $0,3 \mathrm{~mm} /$ dia até chuva maior ou igual a $80,0 \mathrm{~mm} / \mathrm{dia}$. As categorias da chuva estão apresentadas na primeira linha do eixo das abcissas, enquanto o número de observações em cada categoria está na segunda linha. Assim, para o período de 01 a 05/01/2000 existem 104 observações com total pluviométrico diário superior a $0,3 \mathrm{~mm} /$ dia e 11 observações abaixo desse limiar, totalizando 115 dados (23 estações x 5 dias). Ao analisar tais gráficos é importante lembrar que uma previsão perfeita apresenta valores de BIAS e ETS iguais a 1, valores de BIAS inferiores (superiores) a 1 indicam que o modelo está 
subestimando (superestimando) a frequência de ocorrência do evento.

Na Fig. 8a encontram-se os resultados de ETS para as simulações com horizonte de $24 \mathrm{~h}$. Nota-se que o membro $\mathrm{KF}$ apresenta valores superiores aos demais membros, inclusive maior do que a média do conjunto. As melhores previsões, aquelas que apresentam valores mais elevados de ETS, estão nas categorias de chuva entre 10,0 e $50,0 \mathrm{~mm}$, destacando-se a categoria de chuva entre $10,0 \mathrm{e}$ $20,0 \mathrm{~mm}$, onde todos os membros apresentaram valores máximos de ETS. Com o aumento da pluviometria, percebe-se que, de maneira geral, todos os membros têm seu desempenho reduzido.

Quando o tempo de integração é modificado para o horizonte de $48 \mathrm{~h}$ (Fig. 8b), o comportamento é bastante semelhante, com ligeira melhora nas categorias de chuva mais forte. Os melhores resultados, neste caso, são alcançados pela média do conjunto. O membro ZHAO apresenta a melhor performance nas categorias de chuva fraca, destacando-se dos demais, porém sua performance decai em categorias de chuva mais forte. Neste horizonte (48 h), o membro KF apresenta os resultados mais baixos de ETS.
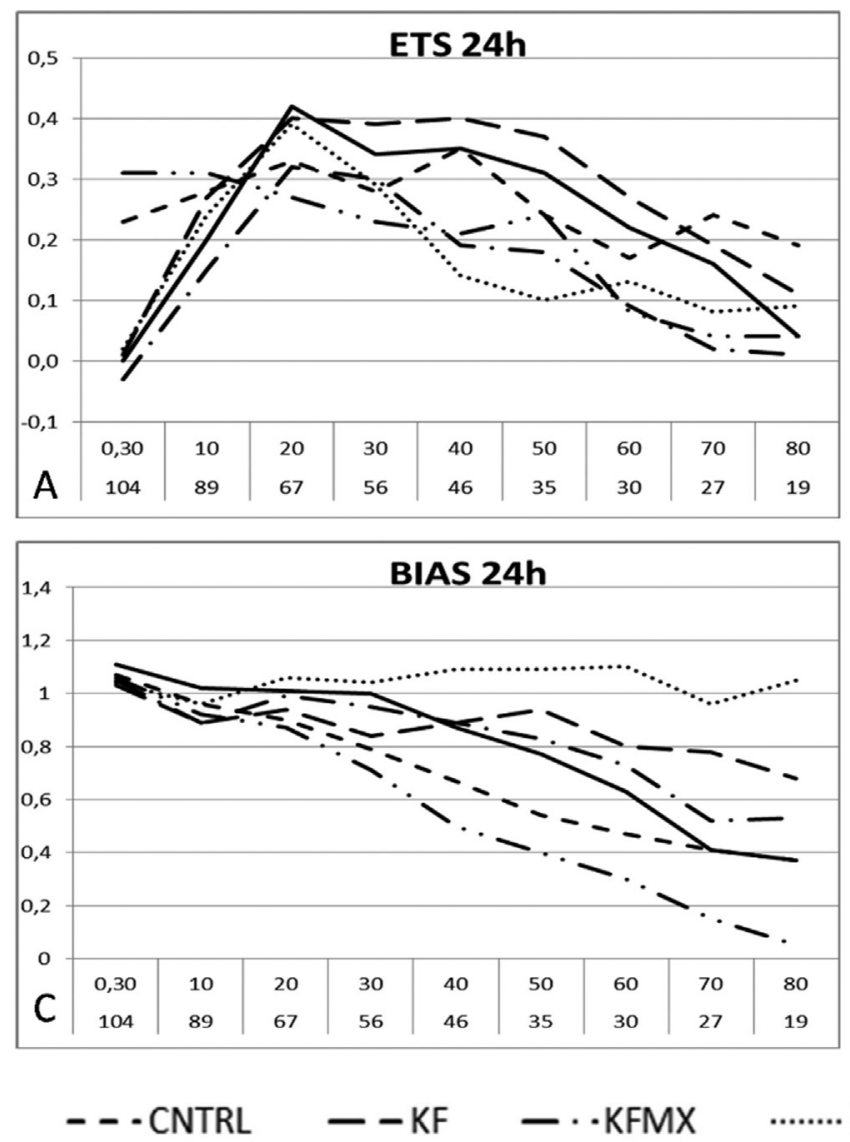

A métrica BIAS pode ser avaliada nas Figs. 8c e 8d. Em ambos os gráficos, categorias com BIAS mais próximo de 1 são aquelas com menores pluviometrias. Conforme o acumulado de chuva vai aumentando, os valores vão discretamente se afastando da unidade. De fato, nota-se que o BIAS varia pouco em relação aos prazos de previsão (24 e $48 \mathrm{~h}$ ), com valores predominantemente menores que 1 nas classes de chuva mais fortes para ambos os gráficos. Destaca-se na Fig. 8c, que o membro KFMXP apresenta valores próximos da unidade ao longo de todas as categorias. Dessa forma, o BIAS indica que esse membro está apresentando melhores resultados que os demais, inclusive com relação à média do conjunto. Nota-se que são apresentados poucos valores de BIAS superiores a 1 , o que indica que há subestimativa geral da chuva em todos os membros (exceto para o membro KFMXP) em todas as categorias. Destaca-se também, maior dispersão entre os membros a partir da categoria de chuvas moderadas, sendo que para o horizonte de $48 \mathrm{~h}$ (Fig. 8d) a dispersão é mais pronunciada. A categoria de chuva acima de $80,0 \mathrm{~mm} / \mathrm{dia}$, em ambos os horizontes, exibe grande dispersão entre os membros. Entretanto, os membros produzem um bom conjunto que apresenta boa consistência em ambos os horizontes.
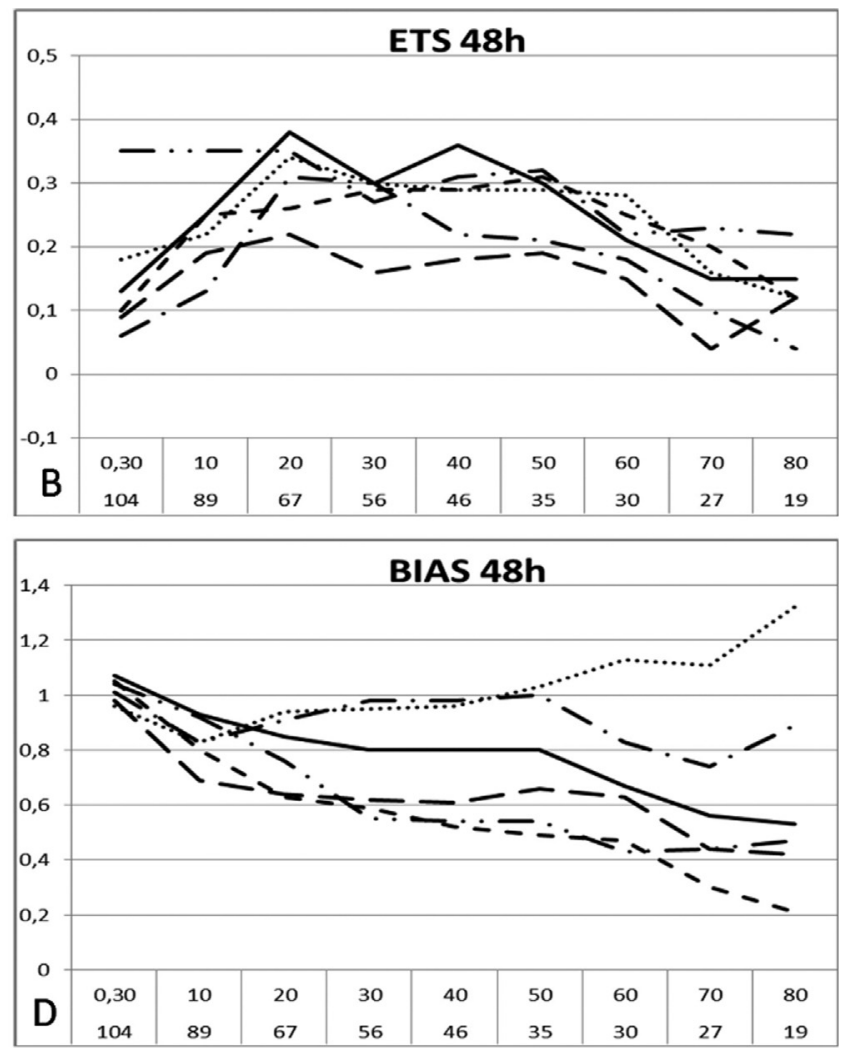

KFMXP - $\cdot$ ZHAO

Média Conjunto

Figura 8 - ETS das simulações elaboradas com 24 h (A) e 48 h (B) de antecedência e BIAS das simulações elaboradas com 24 h (C) e 48 h (D) de antecedência, para os membros CNTRL, KF, KFMX, KFMXP, ZHAO e para a Média do conjunto. Os valores das abscissas referem-se: aos limiares de chuva em mm/dia (na $1^{\mathrm{a}}$ linha) e ao número de observações em cada categoria (na $2^{\mathrm{a}}$ linha). 
Dessa forma, a análise objetiva indica que, especificamente para este estudo de caso, a previsão por conjunto é vantajosa, com os maiores valores de ETS e BIAS também próximos à unidade.

\section{Conclusões}

Neste trabalho realiza-se uma avaliação de uma proposta de sistema de previsão por conjunto do modelo atmosférico Eta com resolução horizontal de $5 \mathrm{~km}$ para um caso de chuva extrema ocorrido em janeiro do ano 2000, na BRPS. O conjunto é formado por quatro membros com diferentes parametrizações convectiva e de microfísica das nuvens (KF, KFMX, KFMXP e ZHAO), além do membro CNTRL. As previsões são avaliadas através dos índices BIAS e ETS, somando-se a uma avaliação subjetiva dos campos de precipitação gerados pelos membros e pela média do conjunto.

Os resultados mostram a grande sensibilidade do modelo à parametrização convectiva para este tipo de evento. Nota-se, da avaliação subjetiva, que os membros que possuem esquema convectivo BMJ (CNTRL e ZHAO) simulam melhor a localização do núcleo de máxima intensidade de chuva. A correta simulação da localização da chuva é de suma importância, de forma a alertar com antecedência os moradores das localidades a serem afetadas, reduzindo-se perdas financeiras e de vidas. Os membros com esquema KF (KF, KFMX e KFMXP) se aproximaram mais do valor máximo acumulado de chuva no dia 03/01/2000.

As simulações geradas pelo conjunto são comparadas às observações através dos parâmetros BIAS e ETS na avaliação objetiva. Os resultados não mostram grandes diferenças entre os valores de BIAS e ETS entre os membros. Para todos os membros, os resultados apresentam decaimento conforme aumenta a intensidade da precipitação, ressaltando a dificuldade na previsão de eventos de chuva intensa e também devido ao menor número de casos para o cálculo dos índices. As simulações que alcançam melhores performances são aquelas para os dias e localidades que apresentaram chuva fraca. De forma geral, as simulações com $48 \mathrm{~h}$ de antecedência são superiores às demais.

Dessa forma, ressalta-se que para este caso a média do conjunto apresentou resultados satisfatórios, contudo é necessário que se faça avaliações de mais casos e de períodos mais longos para se obter um diagnóstico mais detalhado. Assim, será possível implementar novas modificações no modelo de forma a melhorar a performance do conjunto e contribuir ainda mais com a previsão deste tipo de evento na região.

\section{Agradecimentos}

A primeira autora agradece à Coordenação de Aperfeiçoamento de Pessoal de Nível Superior (CAPES) pela bolsa de Mestrado recebida no Programa de Pós-Graduação em Meteorologia (PPGM) da Universidade Federal do Rio de Janeiro (UFRJ). Os autores agradecem ao Conselho Nacional de Desenvolvimento Científico e Tecnológico (CNPq) pelo apoio através do Projeto CNPq/FNDCT No. 400071/2014-2.

\section{Referências}

BRASILIENSE, C.S. Vórtice Ciclônico híbrido embebido na ZCAS associado a um caso de chuva intensa na Bacia do Rio Paraíba do Sul. Dissertação de Mestrado em Meteorologia, UFRJ. pp. 113, 2016.

BRASILIENSE，C.S.; DERECZYNSKI， C.P.; PRAKKI，S.; CHOU, S.C.; RANDER, V.; CALADO, R.N. Synoptic analysis of an intense rainfall event in Paraiba do Sul River Basin in southeast Brazil. Meteorological Applications, v. 25, n. 1, p. 66-77, 2017.

BUSTAMANTE, J.; CHOU, S.C.; ROZANTE, J.R.; GOMES, J.L. Uma avaliação da previsibilidade de tempo do Modelo Eta para a América do Sul. Revista Brasileira de Meteorologia, v. 20, n. 1, p. 59-70, 2005.

BUSTAMANTE, J.; GOMES, J.L.; CHOU, S.C.; ROZANTE, J.R. Evaluation of April 1999 rainfall forecasts over South America using the Eta Model. Climanálise, v. 1, n. 1, p. 17, 1999.

CALVETTI, L. Previsão hidrometeorológica probabilística na Bacia do Alto Iguaçu-PR com os modelos WRF e TopModel. Tese de Doutorado em Meteorologia, USP, pp. 2011. 141, Disponível em: http://www.iag.usp.br/pos/sites/default/files/d_leonardo_calvetti_original.pdf. Acesso em 07/07/2017.

CARVALHO, P.R.B. Inclusão da Perturbação de Momentum no Esquema de Parametrização de Cumulus KainFritsch e Impactos Sobre um Caso de Chuva Convectiva. Dissertação de Mestrado em Meteorologia, INPE, pp. 109, $2007 . \quad$ Disponível em: http://urlib.net/sid.inpe.br/mtc-m17@80/2007/12.07.12.20. Acesso em 07/07/2017.

CHOU, S.C. Modelo regional Eta. Climanálise Edição Especial Comemorativa de $\mathbf{1 0}$ anos, MCT/INPE-CPTEC. v. 10, 1996.

CHOU, S.C.; JUSTI, M.G.A.S. Objective evaluation of Eta Model precipitation forecasts over South America. Climanálise, v. 1, n. 1, 1999.

DOYLE, M.; TOMASELLA J.; RODRIGUEZ, D.A.; CHOU, S.C. Experiments Using New Initial Soil Moisture Conditions and Soil Map in the Eta Model Over La Plata Basin. Meteorology and Atmospheric Physics, v. 121, n. 3, p. 119-136, 2013.

EK, M.; MITCHELL, M.K.; LIU, Y.; ROGERS, E.; GRUNMAN, P.; KOREN, V.; GAYANO, G.;TARPLEY, J. D. Implementation of Noah Land Model advances in the NCEP operational Eta Model. Journal of Geophysical Research, v. 108, n. D22, p. 8851-8867, 2003.

FERRIER, B.S.; JIN, Y.; LIN, Y.; BLACK, T.; ROGERS, E.; DIMEGO, G. Implementation of a new grid-scale cloud and precipitation scheme in the NCEP Eta Model. Conference on Numerical Weather Prediction, 15, San Antonio, TX. American Meteorological Society, p. 280-283, 2002. 
FERRIER, B.S. A double-moment Multiple-phase four-class bulk ice scheme. Part I: Description. Journal of the Atmospheric Sciences, v. 51, n. 2, p. 251-280, 1994.

FESER, F.; ROCKEL, B.; STORCH, H.; WINTERFELDT, J.; ZAHN, M. Regional climate models add value to global model data. A review and selected examples. Bulletin of the American Meteorological Society, v. 92, n. 9, p. 11811192, 2011

GALLUS, W.A.JR. Eta Simulations of Three Extreme Precipitation Events: Sensitivity to Resolution and Convective Parameterization. Weather and Forecasting, v. 14, p. 405426, 1999.

GIORGI, F. Simulation of regional climate using a limited area model nested in a general circulation model. Journal of Climate, v. 3, p. 941-963, 1990.

GIORGI, F. Climate change hot-spots. Geophysical Research Letters, v. 33, p. 1-4, L08707, 2006.

GIORGI, F.; MEARNS, L.O. Approaches to the simulation of regional climate change: A review. Reviews of Geophysics, v. 29, n. 2, p. 191-216, 1991.

GOMES, J.L.; CHOU, S.C. Dependence of partitioning of model implicit and explicit precipitation on horizontal resolution. Meteorology and Atmospheric Physics, v. 106, n. 1-2, p.1-18, 2010.

HAMILL, T.M.; COLUCCI, S.J. Evaluation of Eta-RSM ensemble probabilistic precipitation forecasts. Monthly Weather Review, v. 126, p. 711-724, 1998.

JANJIC, Z.I. The step-mountain Eta coordinate model: Further developments of the convection, viscous sublayer, and turbulence closure schemes. Monthly Weather Review, v. 122 , n. 5, p. 927-945, 1994.

KAIN, J.S. The Kain-Fritsch convective parameterization: An update. Journal of Applied Meteorology, v. 43 p. 170-181, 2004.

KAIN, J.S.; FRITSCH, J.M. Convective parameterization for mesoscale models: the Kain-Fritsch scheme. The representation of Cumulus Convection in Numerical Models. Meteorological Monographs, American Meteorological Society, v. 24, n. 46, p. 165-170, 1993.

LACIS, A.A.; HANSEN, J.E. A parameterization of the absorption of solar radiation in the earth's atmosphere. Journal of the Atmospheric Sciences, v. 31, p. 118-133, 1974.

LOPEZ, P. Cloud and Precipitation Parameterizations in Modeling and Variational Data Assimilation: A Review. Journal of the Atmospheric Sciences - Special Section, v. 64, p. 3766-3784, 2007.

MESINGER, F. Forward-backward scheme, and its use in a limited area model. Atmospheric Physics, v. 50, p. 200-210, 1977.

MESINGER, F. A blocking technique for representation of mountains in atmospheric models. Rivista di Meteorologia Aeronautica, v. 44, p. 195-202, 1984.

MESINGER, F.; BLACK, T.L. On the impact on forecast accuracy of the step-mountain (eta) vs. sigma coordinate. Meteorology and Atmospheric Physics, v. 50, p. 47-60, 1992.

MESINGER, F.; JANJIC, Z.I.; NICKOVIC, S.; GAVRILOV, D.; DEAVEN, D.G. The step-mountain coordinate: Model description and performance for cases of Alpine lee cyclogenesis and for a case of Appalachian redevelopment. Monthly Weather Review, v. 116, p. 1493-1518, 1988.
MESINGER, F.; CHOU, S.C.; GOMES, J.L.; JOVIC, D.; BASTOS, P.; BUSTAMANTE, J.F.; LAZIC, L.; LYRA, A.A.; MORELLI, S.; RISTIC, I.; VELJOVIC, K. An upgraded version of the Eta model. Meteorology and Atmospheric Physics, v. 116, n. 3, p. 63-79, 2012.

MESINGER, F.; VELJOVIC, K. Limited area NWP and regional climate modeling: a test of the relaxation vs Eta lateral boundary conditions. Meteorology and Atmospheric Physics, v. 119 p. 1-16, 2013.

MESINGER, F.; VELJOVIC, K. Eta vs. sigma: review of past results, Gallus-Klemp test, and large-scale wind skill in ensemble experiments. Meteorology and Atmospheric Physics, v 129:153, 2017. DOI 10.1007/s00703-016-0496-3.

MOURA, J.D.O. Efeitos do transporte de momentum convectivo na distribuição de chuva. Dissertação de Mestrado em Meteorologia, INPE, pp. 136, 2016. Disponível em http://urlib.net/8JMKD3MGP3W34P/3LLL7AE. Acesso em 07/07/2017.

PALMER, T.N. Predicting uncertainty in forecasts of weather and climate. Reports on Progress in Physics, v. 63, n. 2, p. 71-116, 2000.

PEEL, S.; WILSON, L.J. A diagnostic verification of the precipitation forecasts produced by the Canadian Ensemble Prediction System. Weather and Forecasting, v. 23, p. 596-616, 1999.

ROZANTE, J.R. Aninhamento do modelo regional Eta sobre América do Sul. Dissertação de mestrado em meteorologia. INPE. pp. 132, 2001. Disponível em: http://mtc-m16.sid.inpe.br/col/sid.inpe.br/jeferson/2004/11. 23.15.00/doc/publicacao.pdf. Acesso em 07/07/2017.

ROZANTE, J.R.; MOREIRA, D.S.; GONÇALVES, L.G.G; VILA, D.A. Combining TRMM and Surface Observations of Precipitation: Technique and Validation over South America. Weather and Forecasting, v. 25, p. 885-894, 2010.

SAHA, S.; MOORTHI, S.; PAN, H-L.; WU, X.; WANG, J.; NADIGA, S.; TRIPP, P.; KISTLER, R.; WOOLLEN, J.; BEHRINGER, D.; et al. The NCEP Climate Forecast System Reanalysis. Bulletin of the American Meteorological Society, v. 91, p. 1015-1057, 2010.

SCHWARZKOPF, M.D.; FELS, S.B. The simplified exchange method revisited: An accurate, rapid method for computation of infrared cooling rates and fluxes. Journal of Geophysical Research, v. 96, p. 9075-9096, 1991.

SIQUEIRA, V.A.; COLLISCHONN, W.; FAN, F.M.; CHOU, S.C. Ensemble flood forecasting based on operational forecasts of the regional Eta EPS in the Taquari-Antas basin. Revista Brasileira de Recursos Hídricos, v. 21, n. 3, p. 587-600, 2016.

SOLMAN, S.A. Regional climate modeling over South America: A review. Advances in Meteorology, v. 2013, p.1-13, 2013.

SUGAHARA, S.; SILVEIRA, R.B.; ROCHA, R.P. Estimativa da Probabilidade do Evento Extremo de Precipitação de Janeiro de 2000 no Vale do Paraíba Baseada na Distribuição Generalizada de Pareto. Revista Brasileira de Geofísica, v. 28, n. 2, p. 193-208, 2010.

TENESSONG, R.S.; VOUNDOU, D.A.; INGRI, P.M.; KAMGA, F.M., Evaluation of Eta weather forecast model over central Africa. Atmospheric and Climate Sciences, v. 2, p. 532$537,2012$. 
TOTH, Z.; KALNAY, E.; TRACTON, S.M.; WOBUS, R.; IRWIN, J. A Synoptic Evaluation of the NCEP Ensemble. Weather and Forecasting, v. 12, p. 140-153, 1997.

WILSON, L.J., BURROWS, W.R., LANZINGER, A.A Strategy for Verification of Weather Element Forecasts from an En- semble Prediction System. Monthly Weather Review, v. 127, p. 956-970, 1999.

ZHAO, Q.; BLACK, T.L.; BALDWIN, M.E. Implementation of the cloud prediction scheme in the Eta Model at NCEP. Weather and Forecasting, v. 12, p. 697-712, 1997.

This is an Open Access article distributed under the terms of the Creative Commons Attribution Non-Commercial License which permits unrestricted non-commercial use, distribution, and reproduction in any medium provided the original work is properly cited. 